\title{
On the topology of stationary black holes
}

\author{
Piotr T. Chruściel ${ }^{* \ddagger}$ \\ Max Planck Institut für Astrophysik \\ Karl Schwarzschild Strasse 1 \\ D 85740 Garching bei München \\ Robert M. Wald ${ }^{\dagger \ddagger}$ \\ Enrico Fermi Institute and Department of Physics \\ University of Chicago \\ 5640 S. Ellis Ave., Chicago, IL 60637.
}

\begin{abstract}
We prove that the domain of outer communication of a stationary, globally hyperbolic spacetime satisfying the null energy condition must be simply connected. Under suitable additional hypotheses, this implies, in particular, that each connected component of a cross-section of the event horizon of a stationary black hole must have spherical topology.
\end{abstract}

\section{Introduction}

The theory of the uniqueness of stationary black holes in classical general relativity intertwines the global techniques of differential geometry with those of the theory of geometric partial differential equations. In spite of considerable progress in the understanding of the issues involved, several open questions in that theory still remain (cf. e.g. [4] for a recent' review). As has been recently stressed by Galloway [ $\mathbb{B}$, one of those is the expected spherical topology of connected components of spacelike sections of event horizons. Recall that such a claim has been made in [12, 11], but, as discussed in detail in [8], the arguments given there do not seem to exclude the possibility of toroidal topology, except perhaps when analyticity up-to-and-including the event horizon is assumed ( $c f .$, however, [10] for a result in the electrovacuum

\footnotetext{
*Alexander von Humboldt fellow. On leave of absence from the Institute of Mathematics, Polish Academy of Sciences, Warsaw. Supported in part by a Polish Research Council grant KBN 2 P302 09506 and by the Federal Ministry of Science and Research, Austria. Current address: Département de Mathématiques, Faculté des Sciences, Parc de Grandmont, F 37200 Tours, France. e-mail: chrusciel@univ-tours.fr

${ }^{\dagger} e-m a i l:$ rmwa@midway.uchicago.edu

${ }^{\ddagger}$ Supported in part by NSF grant PHY-9220644 to University of Chicago.

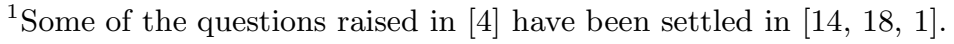


case with non-vanishing charge). While this analyticity seems a plausible property in retrospect, as a potential consequence of the uniqueness theorems, no a priori reasons for analyticity have been given so far. The object of this letter is to point out that toroidal topology of stationary black holes - as well as all other non-spherical topologies - can be excluded as a simple consequence of the "topological censorship theorem" of Friedman, Schleich and Witt [6], when a suitable energy condition is imposed. Moreover the differentiability conditions on the event horizon implicitly assumed in [11] are not needed in our argument to exclude the toroidal, as well as the higher genus topologies.

We shall present the detailed statement of our Theorem in Section 2 below. Before doing that, let us point out that some related results have been proved previously by Gannon [10], by Galloway [8, [7] (cf. also [9]) and by Masood-ul-Alam [17] ( $c f$. also [15]) under various supplementary hypotheses. The proof below arose as a byproduct of an attempt to gain insight into the topology of black holes using the topological censorship theorem of Galloway [8]. A related application of the Friedman-Schleich-Witt topological censorship theorem can be found in $[16$.

\section{The theorem}

We begin by arguing that it suffices to consider the case where the spacetime has a single asymptotically flat region. To make things precise, let $\left(M, g_{a b}\right)$ be a globally hyperbolic space-time with Cauchy surface $\Sigma$ and with a one parameter group of isometries, $\phi_{t}$, generated by Killing vector field $X^{a}$. $\Sigma$ will be assumed at first to have a (possibly infinite) number of asymptotic regions $\Sigma_{i}$, in which $X^{a}$ is timelike and tends asymptotically to a non-zero multiple of the unit normal to $\Sigma$ as the distance away from some fixed point $p \in \Sigma$ tends to infinity. Here the notions of asymptotic flatness and of stationarity are used in the sense of Definitions 2.1 and 2.4 of [5]; we emphasize that $X^{a}$ is not required to be globally timelike. We shall moreover assume that the orbits of $X^{a}$ are complete on $M$. Let us mention that this last hypothesis can be derived as a consequence of field equations and of appropriate hypotheses on $\Sigma$, if field equations are assumed, cf. e.g. [3] and [5, Proposition 3.1].

Consider an asymptotically flat three-end $\Sigma_{i}$, and let $\mathcal{B}_{i}$ and $\mathcal{W}_{i}$ be the black- and white-hole regions with respect to $\Sigma_{i}$ as defined in [5]. Consider the domain of outer communication $\ll \mathcal{J}_{i} \gg$ defined as

$$
\ll \mathcal{J}_{i} \gg=M \backslash\left\{\mathcal{B}_{i} \cup \mathcal{W}_{i}\right\} .
$$

The following result follows immediately from what has been said in [6]:

Proposition 2.1 Under the conditions above, suppose moreover that the null energy condition holds

$$
R_{a b} Y^{a} Y^{b} \geq 0 \text { for all null } Y^{a} .
$$


Then

$$
\ll \mathcal{J}_{i} \gg \cap J^{ \pm}\left(\ll \mathcal{J}_{j} \gg\right)=\emptyset \text { for } i \neq j
$$

In other words, the domain of outer communication associated to the asymptotic three-end $\Sigma_{i}$ is causally separated from those associated to the remaining asymptotic regions. Alternatively, when analysing globally hyperbolic domains of outer communication in which (2.1) holds one can without loss of generality assume that the relevant Cauchy surface has only one asymptotically flat region, as we desired to show. Let us also mention that a somewhat similar result has been proved in [5, Lemmas 3.2 and 3.3]. There it is assumed that the time orientation of the Killing vector in the $i$ 'th end is opposite to that in the $j$ 'th end, but no energy conditions are assumed.

In the following, we shall assume that $\left(M, g_{a b}\right)$ contains a single asymptotically flat region whose domain of outer communications will be denoted by $\ll \mathcal{J} \gg$. We no longer need assume that $M$ is globally hyperbolic, but we require global hyperbolicity of $\ll \mathcal{J} \gg$ (which automatically holds if $M$ is globally hyperbolic). Let $\mathcal{H} \equiv \partial I^{-}(\ll \mathcal{J} \gg)$ denote the future event horizon of the black holes of $M$. For some of our results, we shall assume, in addition, that there exists an achronal, asymptotically flat slice, $\mathcal{S}$, of $\ll \mathcal{J} \gg$ whose boundary in $M$ intersects each null generator of $\mathcal{H}$ at precisely one point. In particular, this implies that the topology of $\mathcal{H}$ is $\mathbf{R} \times K$, where $K=\overline{\mathcal{S}} \cap \mathcal{H}$. (We do not assume that $K$ is connected.) Note that these hypotheses encompass, in particular the case where no black hole is present (in which case $K$ is empty), the case of "spacetimes of class (b)" as defined in [5], as well as "extreme black holes" (such as the Papapetrou-Majumdar solution) not treated in [5].

Let $p$ be any point in the asymptotically flat region of $\ll \mathcal{J} \gg$, so that, in particular, $X^{a}$ is timelike at $p$. Define $\mathcal{C}=\partial I^{+}(p)$. Then $\mathcal{C}$ automatically is an achronal, $C^{1-}$ hypersurface. We write $\mathcal{C}^{\prime}=\ll \mathcal{J} \gg \cap \mathcal{C}, \mathcal{C}_{\text {ext }}=\mathcal{C} \cap M_{\text {ext }}$, with $M_{\text {ext }}$ denoting that part of $M$ which is covered by a single coordinate system in which the metric is asymptotically flat and time-independent, with the Killing vector being timelike there. The proof of our main theorem below will make use of the following lemma:

Lemma 2.2 : Let $\left(M, g_{a b}\right)$ be a stationary, asymptotically flat spacetime containing a single asymptotically flat region whose domain of outer communications, $\ll \mathcal{J} \gg$, is globally hyperbolic. Then

1. Each Killing orbit in $\ll \mathcal{J} \gg$ intersects $\mathcal{C}$ in precisely one point; hence, in particular, $\ll \mathcal{J} \gg$ has topology $\mathbf{R} \times \mathcal{C}^{\prime}$

2. Suppose that there exists an achronal, asymptotically flat slice, $\mathcal{S}$, of $\ll \mathcal{J} \gg$, whose boundary in $M$ intersects the event horizon, $\mathcal{H}$, of any black holes in $M$ in a cross-section, $K$. If $K$ is compact, then each null generator of $\mathcal{H}$ intersects $\mathcal{C}$ in precisely one point; hence, in particular, $\partial \mathcal{C}^{\prime}$ has topology $K$. 
Proof: To prove the first statement, we note that, by the same argument as used in the proof of lemma 3.1 of [5], for any Killing orbit, $\alpha$, in $\ll \mathcal{J} \gg$, we have $\ll \mathcal{J} \gg \subset I^{+}(\alpha)$ and $\ll \mathcal{J} \gg \subset I^{-}(\alpha)$. Consequently, any Killing orbit in $\ll \mathcal{J} \gg$ enters both $I^{+}(p)$ and $I^{-}(p)$, and, thus, must intersect $\mathcal{C}$. If an orbit in $\ll \mathcal{J} \gg$ intersected $\mathcal{C}$ in more than one point, there would exist a $q \in \ll \mathcal{J} \gg$ and a $t>0$ such that both $q$ and $\phi_{t}(q)$ both lie on the boundary of the future of $p$. Equivalently, $q$ lies on the boundary of the future of both $p$ and $\phi_{-t}(p)$. But this is impossible, since $p \in I^{+}\left(\phi_{-t}(p)\right)$.

To prove the second statement, we note first that, by arguments similar to those of the previous paragraph, we may assume without loss of generality that $p \in \mathcal{S}$. By hypothesis, any generator, $\lambda$, of $\mathcal{H}$ intersects $\partial \mathcal{S}$, and, thus, (since $\mathcal{S}$ is achronal) contains a point not lying in $I^{+}(p)$. On the other hand, since $\lambda$ contains a point lying in $\partial \mathcal{S} \subset \partial \ll \mathcal{J} \gg$ and $\lambda$ cannot have a future endpont, it follows that $\lambda$ enters $I^{+}(\ll \mathcal{J} \gg)$. However, $I^{+}(\ll \mathcal{J} \gg) \subset I^{+}\left(\alpha_{p}\right)$, where $\alpha_{p}$ denotes the Killing orbit through $p$. Hence, if we define $K_{t}$ to be the subset of generators of $\mathcal{H}$ which enter $I^{+}\left(\phi_{t}(p)\right)$, we see that $\left\{K_{t}\right\}$ for $t \in \mathbf{R}$ yields an open cover of $K$. By compactness of $K$, there exists a $t_{0} \in \mathbf{R}$ such that every generator of $\mathcal{H}$ enters $I^{+}\left(\phi_{t_{0}}(p)\right)$. Applying $\phi_{-t_{0}}$ to this statement, we conclude that every generator of $\mathcal{H}$ must enter $I^{+}(p)$, and, hence, $\lambda$ must intersect $\mathcal{C}$. Finally, to show that $\lambda$ cannot intersect $\mathcal{C}$ more than once, we note that any $q \in \mathcal{H} \cap \mathcal{C}$ must lie on a null geodesic in $\mathcal{C}$, which, by global hyperbolicity of $\ll \mathcal{J} \gg$, must have a past endpoint on $p$. Hence, if $q, r \in \lambda \cap \mathcal{C}$ with $q \neq r$, one of them would be connected to $p$ by a future-directed broken null geodesic, and, thus, could not lie in $\mathcal{C}$

Our main result is the following:

Theorem 2.3 Let $\left(M, g_{a b}\right)$ be a stationary, asymptotically flat spacetime containing a single asymptotically flat region whose domain of outer communications, $\ll \mathcal{J} \gg$, is globally hyperbolic. Suppose that the null energy condition (2.1) holds. Then

1. $\ll \mathcal{J} \gg$ is simply connected.

2. Suppose that there exists an achronal, asymptotically flat slice, $\mathcal{S}$, of $\ll \mathcal{J} \gg$, whose boundary in $M$ intersects the event horizon, $\mathcal{H}$, of any black holes in $M$ in a cross-section, $K$. If $K$ is compact and if $\mathcal{C}^{\prime} \backslash \mathcal{C}_{\text {ext }}$ has compact closure in $M$ (where $\mathcal{C}, \mathcal{C}_{\text {ext }}$ and $\mathcal{C}^{\prime}$ were defined above), then each connected component of $K$ is homeomorphic to a sphere.

\section{Remarks:}

1. Simple connectedness of $\ll \mathcal{J} \gg$ is equivalent to simple connectedness of any Cauchy surface $\Sigma$ for $\ll \mathcal{J} \gg$. In particular it follows from point 1 of Theorem 2.3 and of Lemma 4.9 of [13] that if $\Sigma$ is homeomorphic to the interior of a compact manifold with boundary $\bar{\Sigma}$, then each connected component of $\partial \bar{\Sigma}$ is homeomorphic to a sphere. 
2. The set $K$ in point 2 above can be empty - in that case we obtain a generalization of the results of [17, 15]; $c f$. Remark 11 above.

3. Recall that a construction of Carter [2] reduces the question of uniqueness of stationary rotating black holes to that of an appropriate harmonic map problem. In that construction simple connectedness of $\ll \mathcal{J} \gg$ plays a key role, compare $[19,20]$.

Proof: Using the first property of Lemma 2.2, we define a continuous time function $\tau$ on $\ll \mathcal{J} \gg$ by the condition that for each $q \in \ll \mathcal{J} \gg$, we have $\phi_{-\tau(q)}(q) \in \mathcal{C}$. It follows immediately that $\tau$ increases monotonically along any future-directed timelike curve, and that for all $q \in \ll \mathcal{J} \gg$ and all $t \in \mathbf{R}$, we have $\tau\left(\phi_{t}(q)\right)=\tau(q)+t$. (A smooth time function on $\ll \mathcal{J} \gg$ with these properties could be obtained by the construction of Proposition 4.1 of [5].) We thereby obtain the identification $\ll \mathcal{J} \gg \approx \mathbf{R} \times \mathcal{C}^{\prime}$ as already noted in Lemma 2.2. To prove point 1 , it suffices to show that any closed path, $\gamma$, in $\mathcal{C}^{\prime}$ is contractible in $\mathcal{C}^{\prime}$. Without loss of generality we may assume that $p \in \gamma$ (where, we recall that $\left.\mathcal{C} \equiv \partial I^{+}(p)\right)$. Without loss of generality we also may assume that $p$ lies on a two-sphere $S^{2} \subset M_{\text {ext }}$ the null inward pointing normals of which are everywhere converging, where, as defined above, $M_{\text {ext }}$ is defined as the orbit of the asymptotically flat end $\Sigma_{\text {ext }}$ under the isometries.

Consider first the simpler case where $X^{a}$ is timelike on $\ll \mathcal{J} \gg$. Let $s \in$ $[0,2 \pi]$ be any parameter on $\gamma$ with $\gamma(0)=\gamma(2 \pi)=p$. By compactness of $S^{1}$ we can choose a constant $A$ large enough so that the curve

$$
[0,2 \pi] \ni s \rightarrow \Gamma(s)=(A s, \gamma(s)) \in \mathbf{R} \times \mathcal{C}^{\prime}
$$

is timelike. The curve $\Gamma$ is then a causal curve from $(0, p)$ to $(2 \pi A, p)$, so that it follows from [6] that $\Gamma$ is homotopic to the curve $\tilde{\Gamma}(s)=(A s, p)$ keeping both end points fixed. Since $\mathcal{C}^{\prime}$ is a deformation retract of $\mathbf{R} \times \mathcal{C}^{\prime}$, contractibility of $\gamma$ in $\mathcal{C}^{\prime}$ follows.

To cover the case in which ergoregions occur some more work is needed. Let thus $p, \gamma$, etc., be as above, and consider any $q \in \gamma$. We first wish to show that there exists a $T \in \mathbf{R}$ and a future directed causal curve from $(0, q)$ to $(T, q)$ the projection of which to $\mathcal{C}^{\prime}$ is homotopically trivial. Indeed, since $(0, q) \in \ll \mathcal{J} \gg$, it follows from Lemma 3.1 of [5] that there exists a future directed timelike curve $\Gamma_{1}(s), s \in[0,1]$, from $\left(T_{1}, p\right)$ to $(0, q)$ for some $T_{1} \in$ R. Similarly there exists a future directed timelike curve $\Gamma_{2}(s), s \in[0,1]$ from $(0, q)$ to $\left(T_{2}, p\right)$ for some $T_{2} \in \mathbf{R}$. The curve $\Gamma_{3}=\phi_{T_{2}-T_{1}}\left(\Gamma_{1}\right) \circ \Gamma_{2}$ is then a future directed timelike curve from $(0, q)$ to $\left(T_{2}-T_{1}, q\right)$. Note that the curve $\Gamma_{2} \circ \Gamma_{1}$ is a causal curve from $\mathbf{R} \times \mathcal{C}^{\prime}$ ext to itself, and hence has homotopically trivial projection on $\mathcal{C}^{\prime}$ by $[6]$. But the projections on $\mathcal{C}^{\prime}$ of $\Gamma_{2} \circ \Gamma_{1}$ and of $\Gamma_{3}$ coincide, which establishes homotopic triviality of $\Gamma_{3}$.

Consider now $q(s) \in \gamma$, denote by $\Gamma_{s}$ the timelike curve from $(0, q(s))$ to $(T(s), q(s))$ just constructed. It is convenient to identify $s \in[0,2 \pi]$ with some parameter on $S^{1}$ in the obvious way. There exists a neighbourhood $\mathcal{O}_{s} \subset \mathcal{C}^{\prime}$ of $q(s)$ such that any two points $r \in\{0\} \times \mathcal{O}_{s}$ and $\tilde{r} \in\{T(s)\} \times \mathcal{O}_{s}$ 
lie on a causal curve which coincides with $\Gamma_{s}$ except near its end points. It follows that there exists $\epsilon(s)>0$ such that for all $s_{-} \in(s-\epsilon(s), s]$ and $s_{+} \in[s, s+\epsilon(s))$ there exists a causal curve $\Gamma_{s_{-}, s_{+}}$between $\left(0, \gamma\left(s_{-}\right)\right)$and $\left(T(s), \gamma\left(s_{+}\right)\right)$. Reducing $\epsilon$ if necessary the projection on $\mathcal{C}^{\prime}$ of $\Gamma_{s_{-}, s_{+}}$can be chosen to be homotopic with both ends fixed to $\left.\gamma\right|_{\left[s_{-}, s_{+}\right]}$.

Consider finally the covering $\{(s-\epsilon(s), s+\epsilon(s))\}_{s \in S^{1}}$ of $S^{1}$. By compactness a finite covering $I_{i}=\left(s_{i}-\epsilon\left(s_{i}\right), s_{i}+\epsilon\left(s_{i}\right)\right), i=1, \ldots, N$ can be chosen. We can order the intervals $I_{i}$ in the obvious way and choose them to be pairwise disjoint except for the nearest neighbours, with $p \in I_{1}$ and $p \in$ $I_{N}$. Let $\gamma\left(r_{0}\right)=p, \gamma\left(r_{N}\right)=p$, and for $1 \leq i \leq N-1$ let $r_{i}$ be any point in $I_{i} \cap I_{i+1}$. By construction for all $i$ there exists a causal curve $\Gamma_{i}$ from $\left(0, \gamma\left(r_{i}\right)\right)$ to $\left(T\left(r_{i}\right), \gamma\left(r_{i+1}\right)\right)$. Moreover the projection of $\Gamma_{i}$ on $\mathcal{C}^{\prime}$ is homotopic with both ends fixed to $\left.\gamma\right|_{\left[r_{i}, r_{i+1}\right]}$. It follows that the curve $\phi_{T\left(r_{1}\right)+\ldots+T\left(r_{N-2}\right)}\left(\Gamma_{N-1}\right) \circ \ldots \circ \phi_{T\left(r_{0}\right)}\left(\Gamma_{1}\right) \circ \Gamma_{0}$ is a causal curve from $\mathbf{R} \times \mathcal{C}^{\prime}$ ext to itself, the projection $\tilde{\gamma}$ of which on $\mathcal{C}^{\prime}$ is homotopic to $\gamma$. By [6] $\tilde{\gamma}$ is homotopically trivial. It follows that $\gamma$ is contractible to $p$, and point 1 follows.

To prove point 2 , we note that the closure in $M$ of $\mathcal{C}^{\prime} \backslash \mathcal{C}_{\text {ext }}$ is a compact manifold with boundary, whose interior is simply connected. Furthermore, by Lemma 2.2, the boundary of this manifold is homeomorphic to the disjoint union of $K$ with a two-sphere in the asymptotic region. The conclusion then follows 20 directly from Lemma 4.9 of [13].

Acknowledgements P.T.C. wishes to thank G. Galloway for many useful discussions and for comments on a previous version of this paper. He is also grateful to the University of Chicago and to the E. Schrödinger Institute in Vienna for hospitality during work on this paper.

\section{References}

[1] R. Beig and P.T. Chruściel, On Killing vectors in asymptotically flat space-times, in preparation.

[2] B. Carter, The general theory of the mechanical, electromagnetic and thermodynamic properties of black holes, General relativity (S.W. Hawking and W. Israel, eds.), Cambridge University Press, Cambridge, 1979, pp. 294-369.

[3] P.T. Chruściel, On completeness of orbits of Killing vector fields, Class. Quantum Grav. 10 (1993), 2091-2101.

[4] _ "No Hair" Theorems - folklore, conjectures, results, Differential Geometry and Mathematical Physics (J. Beem and K.L. Duggal, eds.), American Mathematical Society, Providence, 1994, gr/qc \# 9402032; Garching preprint MPA 792.

\footnotetext{
${ }^{2}$ We are grateful to G. Galloway for pointing this out.
} 
[5] P.T. Chruściel and R.M. Wald, Maximal hypersurfaces in stationary asymptotically flat space-times, Commun. Math. Phys. 163 (1994), 561-604, gr-qc/9304009.

[6] J.L. Friedman, K. Schleich, and D.M. Witt, Topological censorship, Phys. Rev. Lett. 71 (1993), 1486-1489.

[7] G. Galloway, On the topology of black holes, Commun. Math. Phys. 151 (1993), 53-66.

[8] _ Least area tori, black holes and topological censorship, Differential Geometry and Mathematical Physics (J. Beem and K.L. Duggal, eds.), American Mathematical Society, Providence, 1994.

[9] G. Galloway and T. Frankel, Stable minimal surfaces and spatial topology in general relativity, Math. Zeit. 181 (1982), 396-406.

[10] D. Gannon, On the topology of spacelike hypersurfaces, singularities, and black holes, Gen. Rel. Grav. 7 (1976), 219-232.

[11] S. Hawking and G.F.R. Ellis, The large scale structure of space-time, Cambridge University Press, Cambridge, 1973.

[12] S.W. Hawking, Black holes in general relativity, Commun. Math. Phys. 25 (1972), 152-166.

[13] J. Hempel, 3-manifolds, Princeton University Press, Princeton, 1976, Annals of Mathematics Studies No 86.

[14] M. Heusler, On the uniqueness of the Reissner-Nordström solution with electric and magnetic charge, Class. Quantum Grav. 11 (1994), L49L53.

[15] L. Lindblom and D.R. Brill, Comments on the topology of nonsingular stellar models, Essays in General Relatitivy - a Festschrift for A. Taub (F.J. Tipler, ed.), Academic Press, New York, 1980, pp. 13-19.

[16] T. Jacobson and S. Venkataramani, Topology of event horizons and topological censorship, Univ. of Maryland preprint UMDGR-95-019, 1994.

[17] A.K.M. Masood-ul-Alam, The topology of asymptotically euclidean static perfect fluid space-time, Commun. Math. Phys. 108 (1987), 193211.

[18] I. Rácz and R.M. Wald, Extensions of spacetimes with Killing horizons $I I$, in preparation.

[19] G. Weinstein, On rotating black-holes in equilibrium in general relativity, Commun. Pure Appl. Math. XLIII (1990), 903-948.

[20] - The stationary axisymmetric two-body problem in general relativity, Commun. Pure Appl. Math. XLV (1990), 1183-1203. 